\title{
Towards Accessible Touch Interfaces
}

\author{
Tiago Guerreiro \\ Hugo Nicolau Joaquim Jorge \\ IST / Technical University of Lisbon / INESC-ID \\ R. Alves Redol, 9 \\ 1000-029 Lisbon, Portugal \\ +351214233565
}

\{tjvg, hman\}@vimmi.inesc-id.pt, \{jaj, daniel.goncalves@inesc-id.pt\}

\begin{abstract}
Touch screen mobile devices bear the promise of endless leisure, communication, and productivity opportunities to motor-impaired people. Indeed, users with residual capacities in their upper extremities could benefit immensely from a device with no demands regarding strength. However, the precision required to effectively select a target without physical cues creates problems to people with limited motor abilities. Our goal is to thoroughly study mobile touch screen interfaces, their characteristics and parameterizations, thus providing the tools for informed interface design for motor-impaired users. We present an evaluation performed with 15 tetraplegic people that allowed us to understand the factors limiting user performance within a comprehensive set of interaction techniques (Tapping, Crossing, Exiting and Directional Gesturing) and parameterizations (Position, Size and Direction). Our results show that for each technique, accuracy and precision vary across different areas of the screen and directions, in a way that is directly dependent on target size. Overall, Tapping was both the preferred technique and among the most effective. This proves that it is possible to design inclusive unified interfaces for motor-impaired and able-bodied users once the correct parameterization or adaptability is assured.
\end{abstract}

\section{Categories and Subject Descriptors}

H.5.2 [Information Interfaces and Presentation]: User Interfaces - Input devices and strategies, User-centered design.

\section{General Terms}

Performance, Design, Experimentation, Human Factors.

\section{Keywords}

Touch Screen, Tetraplegic, Interaction Techniques, Evaluation, Mobile device.

\section{INTRODUCTION}

Touch screens are increasingly replacing traditional mobile phone keypads. These interfaces offer several advantages over their button-based counterparts. Particularly, they can easily display different interfaces in the same surface (e.g. 12-key keypad, QWERTY keyboard) or adapt to users' preferences and capabilities [5]. Moreover, the ability to directly touch and

Permission to make digital or hard copies of all or part of this work for personal or classroom use is granted without fee provided that copies are not made or distributed for profit or commercial advantage and that copies bear this notice and the full citation on the first page. To copy otherwise, or republish, to post on servers or to redistribute to lists, requires prior specific permission and/or a fee.

ASSETS'10, October 25-27, 2010, Orlando, Florida, USA.

Copyright 2010 ACM 978-1-60558-881-0/10/10...\$10.00

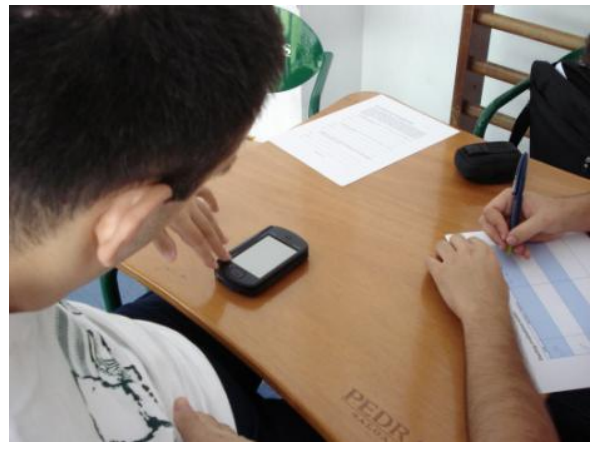

Figure 1 - Motor-impaired user interacting with a mobile touch-screen during evaluation.

manipulate data on the screen without any intermediary device provides a more natural and engaging experience.

However, touch screen interfaces also present challenges for mobile accessibility: they lack both the tactile feedback and physical stability guaranteed by keypads, making it harder for people to accurately select targets. This becomes especially relevant for people who suffer from lack of precision or motor control, such as tetraplegic users.

Still, motor-impaired people could benefit immensely from touch screen devices. Prior work has shown that these users may not have the physical strength, or dexterity, to press hard physical buttons [10]. Additionally, the use of PDAs is a viable alternative to traditional input devices (i.e. mouse and keyboard), allowing the same interface to be used in different places and contexts. Furthermore, the high customization degree of touch screens makes them amenable to custom-tailored or adaptive solutions that better fit each user's needs. This presents good opportunities for motor-impaired people, particularly those who lack both strength and control on their upper limbs. However, it is still very difficult to design better interfaces for this target population as there is no comprehensible knowledge of the values and flaws of each technique in respect to user profiles. Their needs and capabilities have to be taken into account in order to design more effective and efficient mobile touch interfaces.

To overcome this gap we have performed extensive evaluations with 15 tetraplegic people covering four different touch screen interaction techniques (Figure 1). These included Tapping, Crossing targets, and Directional Gesturing. Furthermore we explored both the screen and interface characteristics in search of guidelines for a better user experience.

Our main goal was to provide empirical knowledge to be used in the design of accessible touch interfaces for motor-impaired people. We focused our attention on target sizes and screen locations that are commonly associated with either improved or 
reduced performance (e.g. corners and edges). Only by putting the users first and acknowledging their uniqueness will we be able to design interfaces that can maximize one's performance.

In this paper, we describe our experiment, present and analyze the obtained results for each technique individually, and compare them with each other. Finally, we draw some conclusions and suggestions for future interface designs.

\section{RELATED WORK}

Previous work has tried to improve access to mobile touch screen interfaces by motor-impaired people. Wobbrock et al. [17] proposed a stylus-based approach that uses edges and corners of a reduced touch screen to enable text-entry tasks on a PDA. Results showed that EdgeWrite provides high accuracy and motion stability for users with motor impairments.

Similarly, Barrier Pointing [2] uses screen edges or corners to improve pointing accuracy. By stroking towards the screen barriers and allowing the stylus to press against them, users can select targets with greater physical stability.

Although these works insightfully explore the device physical properties to aid impaired people interacting with touch screens, there is still little empirical knowledge about their performance with other interaction techniques. On the other hand, a great deal of research has been carried out to understand and maximize performance of able-bodied people using these devices $[1,3,6,7$, $8,9,11,12,13]$.

Target size is one of the main issues when studying touch interfaces. The anthropomorphic average width of the index finger and the thumb for adult men are $18.2 \mathrm{~mm}$ and $22.9 \mathrm{~mm}$, respectively, and women $15.5 \mathrm{~mm}$ and $19.1 \mathrm{~mm}$, respectively [1]. HCI literature suggests that for soft buttons to work well with finger interaction, the button width needs to be larger than $22 \mathrm{~mm}$ $[3,7]$. However, while this size is possible to implement in wide screens (e.g. kiosks), they are bigger than what mobile devices accommodate.

Parhi et al. [11] conducted a study to determine optimal target sizes for one-handed thumb use of handheld devices. Results showed that sizes between $9.2 \mathrm{~mm}$ and $9.6 \mathrm{~mm}$ can be used without degrading performance and preference. Similarly, Park et al. [12] analyzed three different virtual key sizes. Results showed that the larger key size $(10 \mathrm{~mm})$ presented higher performance rates and subjective satisfaction. Lee and Zhai [6] obtained similar results, as targets smaller than $10 \mathrm{~mm}$ in width showed strongly reduced performance.

Regarding on-screen target location, users prefer targets near the center of the screen, because it is easier and more comfortable to tap. However, the highest accuracy rates occur for targets on the edge of the screen [12].

The previous studies derive recommendations on target sizes and locations for mobile touch screen interfaces. Mizobuchi et al. [9] conducted a study to determine how text input, using a stylus, would be affected by walking versus standing. They suggest a virtual keyboard with a minimum width of $3 \mathrm{~mm}$ per key, which guarantees an error rate inferior to $2 \%$. However, more demanding walking situations may require larger targets [8]. Users walking in an obstacle course are reported to be able to tap on a $6.4 \mathrm{~mm}$ target with $90 \%$ accuracy.

Although these studies were performed with able-bodied people, with induced impairments [14] and using a stylus, they can reveal
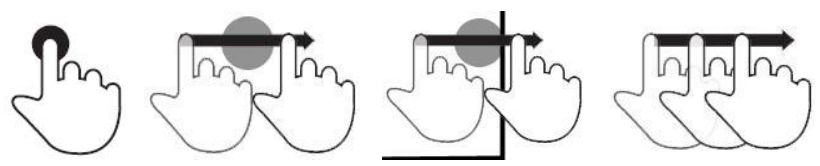

Figure 2 - Tapping, Crossing, Exiting, and Directional Gesturing.

useful insights in the design of touch interfaces for motorimpaired users. Indeed, these users may experience similar problems, as tremor and lack of physical stability. However, the apparent similarities are not enough to assume the results as veritable and the basis for the design of more effective touchbased interfaces for motor-impaired people.

As can be seen, there is a severe lack of results pertaining motorimpaired people when using touch screen devices. The experiment reported in this paper tries to bridge this gap by dissecting interaction techniques, their characteristics and parameterizations, thus providing broader empirical knowledge to support informed touch interface design.

\section{EVALUATING TOUCH TECHNIQUES}

Touch screen devices pose both challenges and opportunities for researchers. Recently, significant efforts have been applied to make these interfaces accessible to motor-impaired people $[2,17]$. Indeed, while they may enable a less physically demanding experience, they are less explored than traditional keypad-based applications. Moreover, to our knowledge, comprehensive studies of touch screen use by these users are yet to be carried out, particularly for tetraplegics with residual arm movement.

Our primary goal with this research was to assess the participants' performance with different interaction techniques, in order to design more effective and efficient touch-based interfaces for motor-impaired people.

\subsection{Interaction Techniques and Variations}

In this experiment, we chose a set of interaction methods representative of the different ways to manipulate a touch interface. This set includes insights from previous work and their assumptions $[2,17]$. We then studied tetraplegic people using those techniques with mobile touch screens.

We considered two basic interaction methods: tapping the screen or performing a gesture. When performing a gesture, users could cross a target, acknowledge a selection by exiting the screen via the intended target, or just use directional gestures (Figure 2).

Tapping the screen consisted in selecting a target by touching it (i.e. land on target). This is the most used interaction technique in current touch screen devices, possibly due to its ease of use or naturalness. In this technique, targets were presented in 3 different sizes $(7,12$, and $17 \mathrm{~mm})$, derived from previous studies for ablebodied users $[6,11,12,13]$, and in all screen positions: edges, corners or middle, thus covering the entire surface.

Crossing, unlike Tapping, did not involve positioning one's finger inside an area. Instead, a target was selected by crossing it. Previous work, on desktop interaction, has shown that this technique offers better performance for motor-impaired users than traditional pointing methods [16]. In our experiment, targets were shown in the middle screen positions (see Figure 3 ) in 3 different sizes.

Exiting was similar to Crossing, but a selection was acknowledged by performing a gesture towards the screen edge 


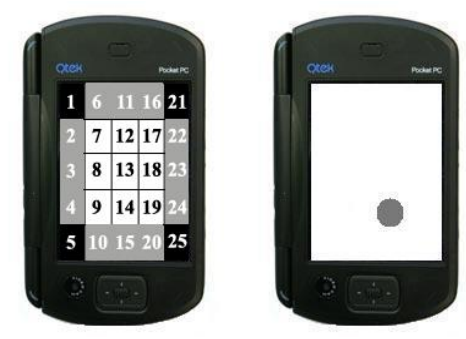

Figure 3 - Qtek 9000. Screen areas (left) :black - corners; gray - edges; white - middle; Target (right).

and crossing the intended target. For this technique targets were shown in all sizes, however, only at edges and corners.

Directional Gesturing was the only technique that did not require a target selection. Users could perform directional gestures anywhere on the device's surface. This technique was chosen both due to its unconstrained nature and, as well as Tapping, because it is a common interaction technique in novel touch-based devices.

Table 1 summarizes all interaction techniques and their variations.

Table 1. Interaction Techniques and Variations

\begin{tabular}{|l|l|l|}
\hline Technique & Sizes & Positions \\
\hline Tapping & $7,12,17 \mathrm{~mm}$ & Middle, Edges, Corners \\
\hline Crossing & $7,12,17 \mathrm{~mm}$ & Middle \\
\hline Exiting & $7,12,17 \mathrm{~mm}$ & Edges, Corners \\
\hline Directional Gesturing & N/A & Middle, Edges \\
\hline
\end{tabular}

\subsection{Research Questions}

This experiment aims to answer several research questions regarding motor-impaired people:

1. What is the best size for each interaction technique?

2. What is the best screen area (e.g. edge, corner or middle) for each interaction technique?

3. What is the best interaction technique?

4. What is the best interaction technique for each screen area?

5. What is the best technique-size-area combination?

\subsection{Participants}

The eight vertebrae in the neck are named cervical vertebrae The top one is called $\mathrm{C} 1$ and the next $\mathrm{C} 2$. Injury of cervical nerves between $\mathrm{C} 1$ and $\mathrm{T} 1$ (first thoracic vertebrae) could result in tetraplegia. Depending on its vertebral level and severity, the individuals with tetraplegia experience a loss of motor and/or sensory functions in their head, neck, shoulders, upper chest, arms, hands and fingers. Injury between $\mathrm{C} 1$ and $\mathrm{C} 4$ is usually called high tetraplegia, while injury between $\mathrm{C} 5$ and $\mathrm{C} 8$ is called low tetraplegia. A person with low tetraplegia may still have partial motor/sensory function in his shoulder, arms, and wrists. Depending on the severity of the injury, individuals may experience complete or incomplete loss of motor/sensory function below the level of injury. In a complete injury, there is no function below the level of the injury.Complete injuries are always bilateral, that is, both sides of the body are affected equally. Incomplete injuries are variable, and a person with such an injury may be able to move one limb more than another, may be able to feel parts of the body that cannot be moved, or may have more functioning on one side of the body than the other.
Fifteen tetraplegic people were recruited from a physical rehabilitation center. The target group was composed by 13 male and 2 female with ages between 28 and 64 years. Table 2 presents user profile information.

Prior to the experiment subjects performed a capability (grasp) assessment test. This functional evaluation aimed to produce a more objective capability identification in opposition to lesion level. However, no correlations between participants' characteristics and task performance were found [4]. Regarding technologic experience, all participants had a mobile phone and used it on a daily basis. However, none of them had a touch screen mobile phone.

\subsection{Apparatus}

In this experiment we used a QTEK 9000 PDA (Figure 3) running Windows Mobile 5.0. The mobile device screen had 640x480 $(73 \times 55 \mathrm{~mm})$ pixels wide, with noticeable physical edges. The evaluation software was developed in C\# using .NET Compact Framework 3.5 and Windows Mobile 5.0 SDK. The evaluation was video recorded and all interactions with the device were logged for posterior analysis.

\subsection{Procedure}

At the beginning of the experiment participants were told that the overall purpose of the study was to investigate and compare different touch interaction techniques and their adequacy for tetraplegic users. We then conducted both a questionnaire and a capability assessment test in order to characterize each participant. Subjects were then informed about the experiment and all interaction techniques (Tapping, Crossing, Exiting, and Directional Gesturing) were explained and demonstrated.

To attenuate learning effects, participants were given warm-up trials before the evaluation of each technique. During these trials they were able to move the mobile device to a comfortable position. All sessions were performed in a quiet environment (their homes or rehabilitation center facilities), always with the participants sitting on their wheelchairs with a table or armrest in front of them (see Figure 1). The interactions with the touch screen were stylus-free; however participants were free to issue selections with any part of their hands/fingers.

Table 2. User profiles.

\begin{tabular}{|l|l|l|l|l|l|}
\hline $\mathbf{P}$ & Age & Gender & Lesion & Type & Hand \\
\hline $\mathbf{1}$ & 28 & Male & C4-C5 & Incomplete & Left \\
\hline $\mathbf{2}$ & 28 & Male & C5 & Complete & Left \\
\hline $\mathbf{3}$ & 29 & Female & C5-C6 & Complete & Right \\
\hline $\mathbf{4}$ & 28 & Female & C5 & Incomplete & Right \\
\hline $\mathbf{5}$ & 61 & Male & C5 & Incomplete & Left \\
\hline $\mathbf{6}$ & 30 & Male & C4-C5 & Complete & Right \\
\hline $\mathbf{7}$ & 34 & Male & C4 & Incomplete & Right \\
\hline $\mathbf{8}$ & 40 & Male & C6-C7 & Complete & Right \\
\hline $\mathbf{9}$ & 61 & Male & C4-C5 & Complete & Right \\
\hline $\mathbf{1 0}$ & 42 & Male & C5-C6 & Complete & Right \\
\hline $\mathbf{1 1}$ & 58 & Male & C5-C6 & Incomplete & Right \\
\hline $\mathbf{1 2}$ & 58 & Male & C4-C5 & Complete & Left \\
\hline $\mathbf{1 3}$ & 44 & Male & C5-C6 & Incomplete & Left \\
\hline $\mathbf{1 4}$ & 64 & Male & C5 & Incomplete & Right \\
\hline $\mathbf{1 5}$ & 27 & Male & C4-C5 & Complete & Left \\
\hline
\end{tabular}


Each subject was asked to perform target selections with each technique (Tapping, Crossing and Exiting). For the Directional Gesturing condition, there were no targets and participants only had to perform a gesture in a particular direction (e.g. north). There were sixteen possible directions, including diagonals and repeated directions with edge support (e.g. north using the right edge as a guideline). For the Tapping condition participants were asked to select targets in all screen positions, as shown in Figure 3 . For the Crossing condition we only used the middle area (9 positions). Exiting, where subjects performed a gesture towards the target leaving the screen, had targets positioned at a corner or an edge, which had to be crossed into.

Participants had one attempt to complete the current trial and were not informed on whether or not it was successful. However, they received feedback that an action was performed. The next target appeared following a two second delay after each action.

For the target acquisition methods three different sizes were used (7, 12, and $17 \mathrm{~mm}$ diameter). We selected tests in a random order to avoid bias associated with experience. In each method-size experience set, target positions were also prompted randomly to counteract order effects. Therefore, each subject performed 142 actions, totaling 2130 actions across all participants. Target sizes were selected taking in consideration the experiments reported for able bodied users $[6,11,12,13]$.

\subsection{Measures}

The measures used in this experiment were obtained through our logging application, which captured all user interactions with the mobile device. The dependent variables were Task Error, Precision, Movement Error, and Movement Time.

For target selection techniques (Tapping, Crossing, and Exiting), Precision was calculated as the minimum distance to the center of the target. For the gesturing condition, Precision corresponded to the average distance to the requested direction axis.

For gestural approaches (Crossing, Exiting and Directional Gesturing) both Movement Time and Error [15] were captured. Movement Time corresponded to the time participants spent touching the screen while performing the gesture. Movement Error consisted in the average absolute deviation from the gesture axis. The difference between Movement Error and Precision is that the former relates to the stability of the movement while the latter relates to the task goal (correct direction or proximity to target).

\subsection{Experimental Design and Analysis}

The experiment varied interaction technique, target size and screen position. We used a within-subjects design, where each participant tested all conditions. For the position analysis, we created two extra factors: Vertical Areas and Distance (see Figure $6)$. The latter reflects the target position in relation to the users' support (level 1 refers to the closest position while level 5 refers to the most distant ones). Although it is visually represented for the right-hand participants, this measure was normalized for both hands.

Shapiro-Wilkinson tests of the observed values for Task Errors, Precision, Movement Error, and Time showed to fit a normal distribution for all interaction techniques. Therefore, a Repeatedmeasures ANOVA was used in further analysis.

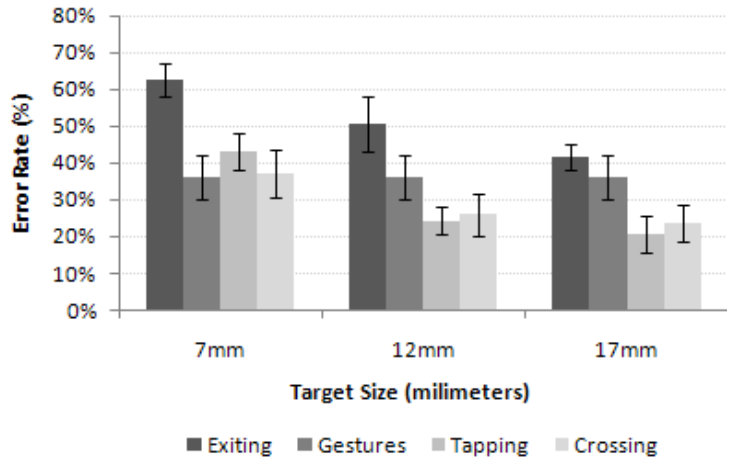

Figure 4 - Task Error Rate for each Technique and Target Size. Error bars denote $95 \%$ confidence intervals.

\section{RESULTS}

Our goal is to understand the capabilities and limitations of the target population relating them with different techniques and outlining their values and flaws. First, we focus on each technique; thus, if they are used in a particular interface, designers can maximize user performance. Second, we analyze the techniques all together and compare their effectiveness in several conditions. This knowledge will enable designers to decide how user actions should be performed in each particular circumstance.

\subsection{Understanding each technique}

The techniques analyzed in this experiment (with overall accuracy results depicted in Figure 4) have different essences and each has its own advantages and disadvantages. In this section, we present the results obtained for each technique and analyze differences strictly within them.

\subsubsection{Tapping}

This technique was analyzed in respect to Task Errors and Precision. Figure 5 presents Task Error Rates for each Target Position across all Target Sizes, offering an overview of Tapping results. Looking at particular experiment variations:

Target Size. There was a significant effect of Target Size on Task Errors $\left(F_{1,42}=25.10, p<.001\right)$. A multiple comparisons post-hoc test found significant differences between small and medium sizes, as well as between small and large sizes (Figure 4). These results suggest $12 \mathrm{~mm}$ as an approximate suitable value for targets to be acquired by motor-impaired users. Regarding Precision, no significant difference was found between target sizes.

Corners. We found no significant effect of Target Position (corner or center) on Task Error Rate (large and medium sizes). A minor effect $\left(F_{1,28}=3.10, \quad p<.1\right)$ was found on the smallest size
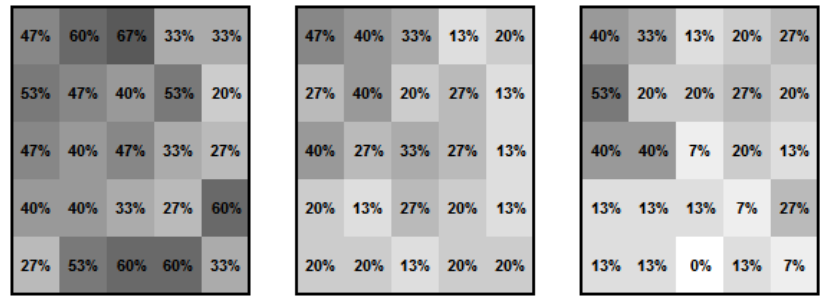

Figure 5 - Task Error Rate heat map for each size: $7 \mathbf{m m}$ (left), $12 \mathrm{~mm}$ (center), and $17 \mathrm{~mm}$ (right). The heat map relates to target center even though targets have different sizes. 


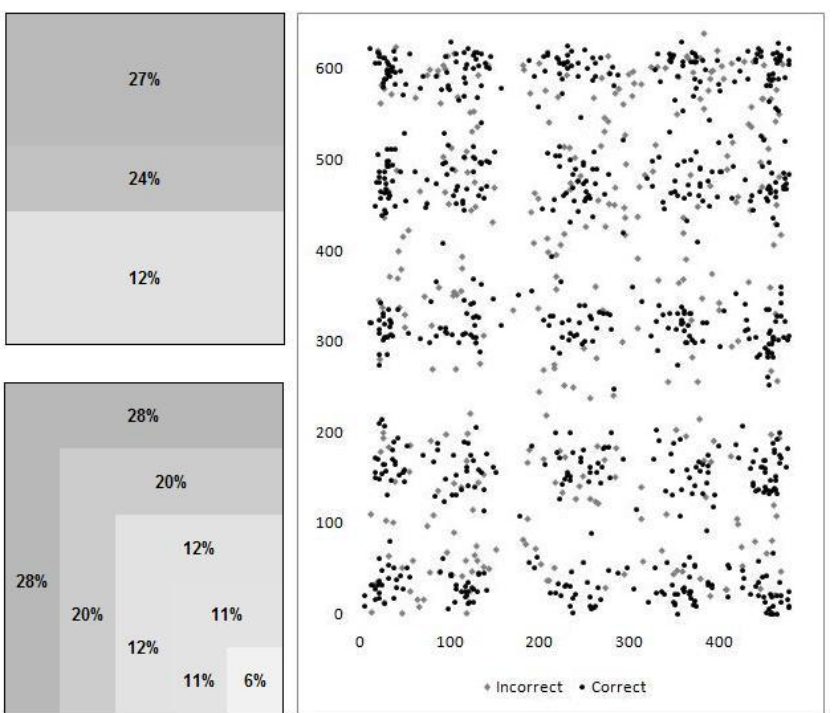

Figure 6 - Task Error Rate (left): vertical areas (top) and distance levels (bottom) in the largest size. Overall taps performed showing both Task Errors and Precision (right).

(less errors in the corners). As to Precision, a significant effect was found on the medium size, pointing to better results in the corners $\left(F_{1,14}=8.941, p<.01\right)$. This indicates that the corners offer higher stability towards a precise movement although this is not reflected in higher accuracy.

Edges. Considering edges, there was no significant difference on Task Errors for Tapping, regardless of target size, or its position on an edge or not. However, there was a significant effect of Target Position (edge or center) on Precision for the smallest $\left(F_{1,28}=14.41, \quad p<.01\right)$, medium $\left(F_{1,28}=6.85, p<.005\right)$ and large $\left(F_{1,28}=27.67, p<.001\right)$ sizes, showing higher precision in the edges. As with corner targets, the physical stability provided by the edges is also beneficial for a more precise tap.

Vertical Areas. We found a significant difference both on medium $\left(\mathrm{F}_{1,42}=3.59, \mathrm{p}<.05\right)$ and largest $\left(\mathrm{F}_{1,42}=5.19, \mathrm{p}<.05\right)$ sizes (Figure 6). Post-hoc tests showed differences to be significant between bottom and top areas, with higher error rates in the top targets. As to Precision, a minor effect was found in the medium and largest sizes, also pointing to differences between top and bottom areas (higher precision in bottom areas). This strongly suggests that the users are more accurate and precise acquiring targets closer to their arm support point.

Distance. Building on the results above, the Target Position (Distance) (Figure 6) had a significant effect on Task Errors in the largest size $\left(F_{1,70}=4.56, \mathrm{p}<.01\right)$. Post-hoc tests showed differences to be significant between levels 1, 2 (closer to users' arm) and level 5. Also, there was a significant effect of Distance on Precision in the smallest size $\left(F_{1,70}=6.04, p<.001\right)$ between levels 2,4 and level 5 , and both medium $\left(F_{1,70}=4.85, p<.01\right)$ and largest size $\left(F_{1,70}=8.31, p<.001\right)$ between levels $2,3,4$ and level 5 .

\subsubsection{Crossing}

This experiment featured targets in the nine (9) central positions, thus avoiding targets close to the edge or corner (those were evaluated individually as a particular technique - Exiting). Crossing included, besides Task Errors and Precision, analysis to Time and Movement Error.

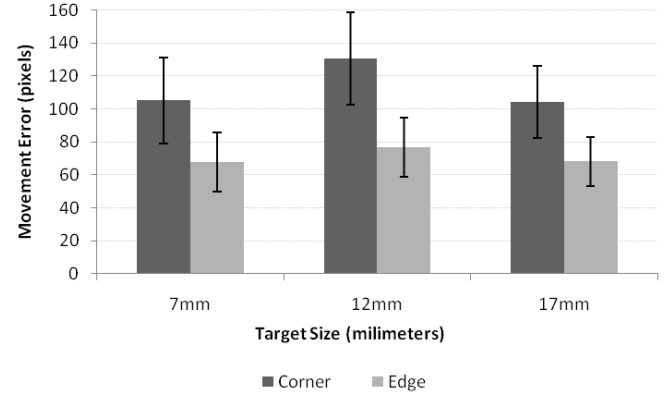

Figure 7 -Movement Error (average) for each Target Size comparing targets in the edges and in the corners (Exiting). Error bars denote $95 \%$ confidence intervals.

Target Size. There was a significant effect of Target Size on Task Errors $\left(F_{1,42}=6.56, p<.01\right)$. Significant differences were found between the smallest and largest sizes (Figure 4). Regarding Precision, a minor effect was also found $\left(F_{1,42}=2.69, p<.01\right)$ between the same sizes. No effect was found in Time or Movement Error. The absence of significant effects suggests that Target Size does not have an influence on the way the users cross the targets (the type of movement and time dispended to accomplish the task).

Vertical Areas. Vertically, no significant effect was found of Target Position (Vertical areas) on Task Errors, Precision, Time or Movement Error. This comes as no surprise as all targets were placed in a center position, minimizing the vertical differences.

Distance. No significant effect was found of Target Position (Distance) on Task Errors, Precision, Time or Movement Error.

\subsubsection{Exiting}

Exiting is similar to Crossing but it is performed with targets on corners and edges. Thus, the users are only able to go towards the target and exit the screen. Exiting included analysis of Task Errors, Precision, Time and Movement Error.

Target size. There was a significant effect of Target Size on Task Errors $\left(F_{1,42}=7.77, p<.01\right)$. Significant differences were found between the smallest and largest sizes (Figure 4). No effect was found in Precision, Time or Movement Error. As with Crossing, the absence of significant effects suggests that Target Size does not have an influence on the way the users perform gestures.

Corners. We found no significant effect of Target Position (corner or just edge) on Task Errors, Time or Precision in any target size. As to Movement Error, a significant effect was found on the smallest $\left(F_{1,28}=5.04, p<.05\right)$, medium $\left(F_{1,28}=9.48, p<.01\right)$ and largest $\left(F_{1,28}=10.32, p<.01\right)$ sizes (Figure 7$)$. Hence, users achieve similar accuracy on corners and edges but produce more erroneous gestures when their movement is restricted (for a target in the corner, the direction of the movement is restricted to 90 degrees, while for an edge the user is restricted to 180 degrees). Observation showed that the users had individual preferences for particular gestures and directions. These preferences may collide with the restriction imposed by a corner target producing the aforementioned erroneous movement.

Vertical Areas. There was a significant effect of Target Position on Task Errors in the medium $\left(F_{1,42}=3.42, p<.05\right)$ size, with higher accuracy in the top targets. Interestingly, a significant effect of Target Position was found both on Precision $\left(F_{1,42}=4.43\right.$, $p<.05)$ and Movement Error $\left(F_{1,42}=4.12, p<.05\right)$, in the smallest size, suggesting that the users were able to counteract the lack of 


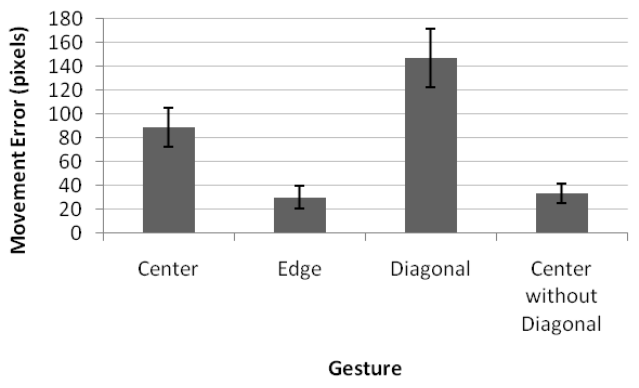

Figure 8 - Movement Error (average) regarding different gesture directions and onscreen areas. Error bars denote $95 \%$ confidence intervals.

accuracy with a more convoluted movement. In the largest size, no significant effects were found. This results contrast with the ones achieved with Tapping. Observation showed that even for top targets, in the majority of the successful cases, the contact with the surface is also performed close to the user (in the bottom) and then the gesture is performed towards the target. This may indicate that users have a better approach with the surface close to them but are then able to produce accurate gestures towards the top (Figure 10 - left and center).

Distance. A significant effect of Target Position (Distance) was found on Precision in the smallest size $\left(F_{1,70}=4.08, p<.05\right)$. This difference was found to be significant between the intermediate distance (worst precision) and both the closest and distant targets. Observation suggested that users tap the surface more accurately the closest to the arm support point (this idea is supported by the aforementioned Tapping results). Upon contact, the most accurate gestures were those with a straighter direction. This is also supported by the differences reported in Vertical areas. A significant effect of Target Position (Distance) was also found on Time both in the medium $\left(F_{1,70}=3.48, p<.05\right)$ and largest $\left(F_{1,70}=3.55, p<.05\right)$ sizes suggesting that the farther are the targets, the longer it takes to acquire them (implies longer gestures as the first contact is preferably performed close to the arm support).

\subsubsection{Directional Gesturing}

As to Directional Gesturing, there are no particular on-screen targets or sizes, just directions. This method included analysis of Task Errors, Precision, Time and Movement Error.

Edges. No significant effect was found between Target Position (gestures supported by the edges or anywhere else on-screen) and Task Errors or Precision. However, a significant effect was found on Movement Error $\left(F_{1,28}=26.68, p<.001\right)$ (Figure 8). This effect was found between gestures performed in the edges (less erroneous) and gestures performed anywhere else on-screen. Nevertheless, these differences are influenced by diagonal directions which are harder to accomplish (and significantly worse than vertical or horizontal gestures). If those are discarded, there are no significant differences between gestures in the "middle of the screen" and supported by edges. As to Time, a significant effect was found between the edge-supported gestures (faster) and both the middle ones $\left(F_{1,70}=2.52, p<.05\right)$.

Vertical Areas. No significant effects were found in any of the dependent variables between vertical areas.

Direction. No significant effect was found between Gesture Direction and Task Errors or Precision. Several errors were due to undesired taps but with no relation with particular directions.
Visual inspection suggested that some directions are more problematic but these differences were not significant. Regarding Movement Error $\left(F_{1,224}=15.14, p<.001\right)$ and Time $\left(F_{1,224}=2.52\right.$, $p<.05)$, a significant effect was found. Figure 9 depicts Movement Error results for all the directions, detailing what was already visible in Figure 8: diagonal gestures are the most erroneous; with two exceptions, gestures supported by the edges are straighter; middle (without diagonals) gestures stand in between.

\subsection{Mixing techniques}

The analysis performed for each technique reinforces the idea that user effectiveness and efficiency is affected by target characteristics like size or on-screen position. This effect has different proportions for the different proposed approaches. We have already addressed each method in this regard. We will now focus on comparing techniques and understanding which is best suited for particular target size/position combinations

\subsubsection{An overview of the techniques}

Figure 4 presents the Task Error Rates for all techniques and sizes. There was a significant effect of Technique on Task Errors in the smallest $\left(F_{1,56}=5.97, p<.01\right)$, medium $\left(F_{1,56}=5.66, p<.01\right)$ and largest $\left(F_{1,56}=8.04, p<.01\right)$ sizes. For the smallest size, the differences are significant between Exiting (worse) and all the others, while for the other sizes, Exiting is significantly worse than Tapping and Crossing. Overall, Exiting stands out as a less accurate method although its flaws diminish, when compared with gesture approaches, as target size increases. Tapping and Crossing were revealed as the most accurate techniques with no significant differences among each other. Overall, Task Errors differences from the medium to the largest size are not significant, suggesting both convergence and the medium size as a good compromise.

\subsubsection{Interacting with edge support}

One can argue that screen edges offer a positive support for interaction. In the techniques considered, the users were asked to tap targets near an edge (Tapping) and to perform gestures with edge support (Directional Gesturing). No significant effect of Interaction Technique (Tapping in the edge vs Gesturing in the edge) was found on Task Errors in the smallest or medium sizes. A minor effect was found in the largest size suggesting better accuracy in edge-supported taps $\left(F_{1,28}=3.15, p<.1\right)$. This is understandable as the edge forces the user to perform the movement in a particular direction, one that may or may not be possible/easy for him to perform. Tapping is less restrictive as the user may approach the target as he is more comfortable to do so.

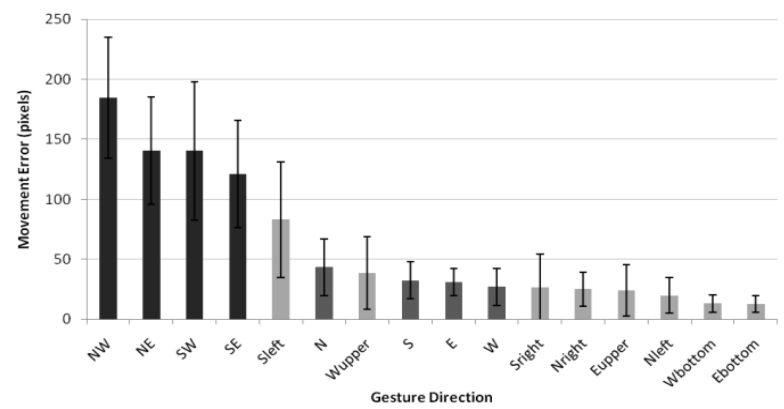

Figure 9-Movement Error (average) for all directional gestures. Darker bars are diagonal gestures, intermediate are other middle gestures, and lighter bars are gestures supported by the edge (e.g., Wupper is a gesture to the West performed in the upper edge). Error bars denote $95 \%$ confidence intervals. 


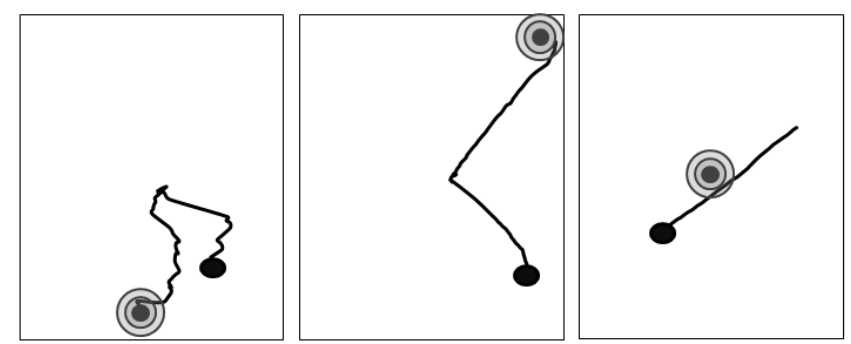

Figure 10 - Example successful gestures performed by Participant \#3. Exiting towards a bottom edge target (left) and a top corner target (center). Crossing a middle target (right).

\subsubsection{Selecting edge targets}

One of the supposed barriers to an effective touch screen experience is the lack of relief supporting visual elements. Commonly, mobile user interfaces present targets near the edge (e.g., close window, shortcuts). The screen edges represent the lasting tactile support (and are vanishing in the latest models, e.g., iPhone). We want to understand if edges are beneficial when acquiring a target and which is the best technique to adopt.

From the presented set of techniques, users selected edge targets by Tapping them or by crossing them towards the screen barrier (Exiting). No significant difference was found between approaches to select edge targets, suggesting similar accuracy.

Also, individual techniques did not show any effect of Target Position (edge or not) on target acquisition accuracy (Task Errors) (refer to 4.1, individual technique analysis). However, a significant effect on Task Errors was found when comparing Crossing and Exiting approaches, for the smallest $\left(F_{1,28}=12.62\right.$, $p<.01)$, medium $\left(F_{1,28}=12.97, p<.01\right)$ and largest $\left(F_{1,28}=5.95\right.$, $p<.05)$ sizes, showing that gesturing towards a mid-screen target is more accurate than towards an edge target (Figure 10). This may be due to direction restrictions imposed in the edge scenario.

\subsubsection{Interacting in the middle of the screen}

The "middle of the screen" refers to all areas away from edges. This represents a major percentage of the interaction surface and it is worthy to comprehend how a user can interact therein. In this experiment, the participants could tap or cross a target and perform directional gestures in the middle of the screen. In this analysis we have discarded diagonal gestures as they were seen as drastically decreasing the success of Directional Gesturing approach. There was no significant effect of Interaction Technique on Task Errors, suggesting that users have similar accuracy while interacting in the middle of the screen with Tapping, Crossing and Directional Gesturing.

\subsubsection{User opinions}

When asked about the techniques' Ease of Use (using a 5-point Likert scale), the median [quartiles] attributed by the users was for Exiting 2[2, 3], for Tapping 4[4, 4.5], for Crossing 4[4, 4] and for Directional Gesturing 4[4, 4], showing a slight preference for Tapping. This idea was reinforced when the users were asked about their preferred method (9/15 selected Tapping, 3/15 selected Crossing, and 3/15 selected Directional Gesturing).

\section{DISCUSSION}

After analyzing each technique in detail, and comparing them with each other, we are now able to answer the research questions proposed at the beginning of this study.

1. What is the best size for each interaction technique?
For all interaction techniques the best sizes are the medium and larger ones (12 and $17 \mathrm{~mm}$ ), with no significant effect on Task Errors between them. From a designer point of view, targets with $12 \mathrm{~mm}$ are therefore, a better commitment due to mobile devices' space constraints. Exception has to be made for the Exiting technique, which has only shown a significant effect between the larger and smaller sizes. Thus, gesturing towards and edge target requires bigger targets. Despite these results, Task Error Rate is still high (20\%), when compared to able-bodied performance reports $[6,11,12,13]$ (lower than 10\%). Thus, future research is needed in order to improve motor-impaired performance when interacting with touch screen devices.

2. What is the best screen area (e.g. edge, corner or middle) for each interaction technique?

Regarding Task Errors, screen areas did not affect users' performance. However, corners and edges allow users to tap targets more precisely, and perform directional gestures less erroneously. This suggests that support from screen barriers, indeed, offers physical stability [2, 17].

Still, when placing targets near edges or corners one has to be careful selecting their size and interaction technique, due to physical restrictions imposed by the device. Although screen barriers offer physical stability, they can also restrict users' movements. For instance, results have shown that acquiring midscreen targets (Crossing) is easier than towards screen barriers (Exiting).

3. What is the best interaction technique for motor-impaired people?

In this study, Tapping and Crossing have shown to be the more effective interaction techniques. However, taking into account users' opinions, Tapping has a slight advantage, as 9/15 selected it as their preferred method.

\section{What is the best interaction technique for each screen area?}

In the middle of the screen neither of the interaction techniques Tapping, Crossing, or Directional Gesturing - was revealed as significantly more accurate. This suggests that users can achieve similar performances with any of them. Nevertheless, when allowing for Directional Gestures, only four directions (North, South, East, and West) should be considered, as diagonals are harder to accomplish by motor-impaired users. Indeed, if those are discarded, Directional Gesturing in the main directions will be even more accurate as the error margin may increase. Correspondingly, at the screen corners, Tapping and Exiting have similar target acquisition performances. Regarding Edges, Tapping is a better choice, since performing a particular Directional Gesture at this screen area may be difficult for tetraplegic users.

\section{What is the best technique-size-area combination?}

Taking into consideration the aforementioned, summing up all research questions, we can conclude that the best combination of technique and size, is Tapping for the medium size $(12 \mathrm{~mm})$. Regarding the area, no significant differences were found on effectiveness but users were more precise tapping edge targets. This combination has shown the best results for tetraplegic users interacting with touch screens. However, particular applications and interfaces may require particular measures. When Tapping is unfeasible or insufficient, results herein presented give space for suitable alternatives. 


\section{CONCLUSIONS}

Touch screen interfaces have shown to be a compelling alternative to traditional button-based interfaces, particularly for those who lack both strength and dexterity on their upper limbs [10]. Nevertheless, these devices impose new challenges to these users, for instance, the absence of physical stability, making it harder for people to accurately select targets.

Some interaction techniques were developed to improve access to mobile touch screens interfaces by motor-impaired people [2, 17]. However, those were built without empirical background and characterization of users' capabilities and needs when interacting with these devices. A step back is required to understand user's capabilities and needs in respect to interfaces' values and flaws.

We undertook an extensive evaluation with 15 tetraplegic users and a set of mobile touch screen interaction techniques, in order to provide empirical knowledge to be used in the design of future interfaces. Our results indicate that users benefit from a better understanding of their capabilities and challenges, particularly considering interaction method (Tapping, Crossing, Exiting, or Directional Gesturing), target size $(7,12$, or $17 \mathrm{~mm})$ and screen area (middle, edges, corners, or proximity to the user).

Overall, Tapping has shown to be a promising interaction technique when compared to alternative methods, such as Crossing or Directional Gesturing. Besides being the preferred technique, users were equally effective with this method as with custom-made techniques. This result suggests that a unified user interface can be developed for both able-bodied and motorimpaired people, with minor adjustments.

Regarding target size, $12 \mathrm{~mm}$ has shown to be the most appropriate diameter. Still, only three target sizes were studied (7, 12 , and $17 \mathrm{~mm}$ ). It is important to recognize that additional research is necessary to refine the set of possible target sizes.

Concerning screen areas, our results confirm that edges and corners offer physical support and stability, allowing users to select target more precisely. However, we did not found an effect on accuracy. Nevertheless, there is an effect of Distance on Error Rate, indicating that users can easily tap (land-on) targets near their support. However, with gesture techniques this effect fades, suggesting that they can acquire more distant targets with support from the screen surface, while adjusting their movement.

\section{ACKNOWLEDGMENTS}

We thank all the users that participated in the studies and João Martins for developing the evaluation application. This work was supported by FCT through the PIDDAC Program funds. Hugo Nicolau and Tiago Guerreiro were supported by FCT, grants SFRH/BD/46748/2008 and SFRH/BD/28110/2006, respectively.

\section{REFERENCES}

[1] Dreyfuss, H. and Alvin, R. and others. 1993. The Measure of Man and Woman. Whitney Library of Design, New York.

[2] Froehlich, J., Wobbrock, J., and Kane, K. 2007. Barrier pointing: using physical edges to assist target acquisition on mobile device touch screens. In Proc of ASSETS '07. ACM Press, New York, 19-26.

[3] Greenstein, J. 1997. Pointing Devices. In Handbook of Human-Computer Interaction, 1317-1348.
[4] Guerreiro, T., Nicolau, H., Jorge, J., Gonçalves, D. 2010. Assessing mobile touch interfaces for tetraplegics. In Proc. of MobileHCI'10. ACM Press, New York.

[5] Kane, S.K., Wobbrock, J.O., and Smith, I.E. 2008. Getting off the treadmill: evaluating walking user interfaces for mobile devices in public spaces. In Proc. of MobileHCI'08 . ACM Press, New York, NY, 109-118.

[6] Lee, S., and Zhai, S. 2009. The performance of touch screen soft buttons. In Proc of CHI'09. ACM Press, New York, NY, 309-318.

[7] Lewis, J. 1992. Literature review of touch screen research from 1980 to 1992. IBM, Boca Raton, FL.

[8] Lin, M., Goldman, R., Price, K., Sears, A., and Jacko, J. 2007. How do people tap when walking? An empirical investigation of nomadic data entry. In International Journal of Human-Computer Studies. Elsevier, 65, 9, 759-769.

[9] Mizobuchi, S., Chignell, M., and Newton, D. 2005. Mobile text entry: relationship between walking speed and text input task difficulty. In Proc. of MobileHCI'05. ACM Press, New York, NY, 122-128.

[10] Myers, B., Wobbrock, J., Yang, S., Yeung, B., Nichols, J., and Miller, R. 2002. Using handhelds to help people with motor impairments. In Proc. of ASSETS '02. ACM Press, New York, NY, 89-96.

[11] Parhi, P., Karlson, A., and Bederson, B. 2006. Target size study for one-handed thumb use on small touchscreen devices. In Proc. of MobileHCI'06 . ACM Press, New York, NY, 203-210.

[12] Park, Y., Han, S., Park, J., and Cho, Y. 2008. Touch key design for target selection on a mobile phone. In Proc. of MobileHCI'08. ACM Press, New York, NY, 423-426.

[13] Perry, K., and Hourcade, J. 2008. Evaluating one handed thumb tapping on mobile touchscreen devices. In Proc. of Graphics Interface. GI'08, Toronto, Canada, 57-64.

[14] Sears, A., Lin, M., Jacko, J., and Xiao, Y. 2003. When computers fade: Pervasive computing and situationally induced impairments and disabilities. In HCI Int'l. Lawrence Erlbaum Associates, 2, 1298-1302.

[15] Soukoreff, R., and MacKenzie, I. 2004. Towards a standard for pointing device evaluation, perspectives on 27 years of Fitts' law research in HCI. In International Journal of Human-Computer Studies. Elsevier, 61, 6, 751-789.

[16] Wobbrock, J., and Gajos, K.. 2008. Goal crossing with mice and trackballs for people with motor impairments: Performance, submovements, and design directions. In ACM Transactions on Accessible Computing (TACCESS), 1, 1. ACM Press, New York, NY, 1-37.

[17] Wobbrock, J., Myers, B., and Kembel, J. 2003. EdgeWrite: a stylus-based text entry method designed for high accuracy and stability of motion. In Proc. of the 16th Annual ACM Symposium on User interface Software and Technology. UIST '03. ACM Press, New York, NY, 61-70. 